\title{
The effectiveness of a stratified care model for non-specific low back pain in Danish primary care compared to current practice: study protocol of a randomised controlled trial
}

Lars Morso ${ }^{1,2^{*}}$ (D) Berit Schiøttz-Christensen ${ }^{2,3}$, Jens Søndergaard ${ }^{4}$, Nils-Bo de Vos Andersen ${ }^{5}$, Flemming Pedersen ${ }^{6}$, Kim Rose Olsen ${ }^{7}$, Morten Sall Jensen ${ }^{1}$, Jonathan Hill ${ }^{8}$ and David Høyrup Christiansen ${ }^{5,9}$

\begin{abstract}
Background: Prior studies indicate that stratified care for low back pain results in better clinical outcome and reduced costs in healthcare compared to current practice. Stratified care may be associated with clinical benefits for patients with low back pain at a lower cost, but evidence is sparse. Hence this study aims to evaluate the clinical effects and cost-effectiveness of stratified care in patients with non-specific low back pain compared to current practice.

Methods/design: The study is a two-armed randomised controlled trial in primary care in the Regions of Southern and Central Denmark (2.5 million citizens). Patients with non-specific low back will be recruited by paticpating GPs. Patients are randomised to either (1) stratified care or (2) current practice at participating physiotherapy clinics. In the stratified care arm, the intervention is based on the patient's STarT Back Tool classification and trained accordingly, whereas physiotherapists in the current pratice arm are blinded to the STarT score. Primary outcomes in the trial will be group differences in time off work, improvement in LBP disability measured by the Roland Morris Disability Questionnaire (RMDQ) and patient-reported global change. Secondary measures will be pain intensity, patient satisfaction, data on patient healthcare resource utilisation and quality-adjusted life year based on the EQ-5D-5L.

Discussion: Stratified care that effectively targets treatment to relevant sub-groups of patients has potentially great impact on the treatment pathways of low back pain. Thus, if effective, this could result in better patient outcomes and at the same time reduce the costs for treatment of low back pain.
\end{abstract}

Trial registration: ClinicalTrials.gov, NCT02612467. Registered on 16 November 2015.

Keywords: Stratified care, STarT back tool, Randomised controlled trial, Cost effectiveness

\section{Background}

Emerging evidence indicates that stratified care of low back pain (LBP) may result in better clinical outcome and reduced healthcare costs, compared to usual care [1]. Stratified care is a way to manage the complexity of

\footnotetext{
* Correspondence: lars.morsoe@rsyd.dk

${ }^{1}$ Centre for Quality, Region of Southern Denmark, P.V. Tuxensvej 5, 5500 Middelfart, Denmark

${ }^{2}$ Department of Regional Health Research, University of Southern Denmark, Odense, Denmark

Full list of author information is available at the end of the article
}

non-specific LBP (NSLBP) [2]. This approach involves a simple nine-item screening questionnaire, the STarT Back Tool (SBT). The SBT has been translated and cross-culturally validated in a Danish-speaking population $[3,4]$. Using this tool, patients are assigned to one of three subgroups, based on modifiable indicators of prolonged LBP, and then manage according to a matched treatment pathway [5].

Patients are classified into low-risk, medium-risk and high-risk subgroups based on the SBT score. Conceptually low-risk patients are likely to have a good outcome

(c) The Author(s). 2018 Open Access This article is distributed under the terms of the Creative Commons Attribution 4.0 International License (http://creativecommons.org/licenses/by/4.0/), which permits unrestricted use, distribution, and reproduction in any medium, provided you give appropriate credit to the original author(s) and the source, provide a link to the Creative Commons license, and indicate if changes were made. The Creative Commons Public Domain Dedication waiver (http://creativecommons.org/publicdomain/zero/1.0/) applies to the data made available in this article, unless otherwise stated. 
with a one-off good quality consultation consisting of reassurance, analgesia and high-quality health information about good self-management. Patients at medium risk are likely to benefit from evidence-based physiotherapy treatment focusing on reducing pain and disability and enabling patients to manage ongoing or future episodes of LBP. High-risk patients are to receive a more comprehensive combined physical and psychosocial intervention, according to this stratified care model termed as psychologically informed physiotherapy (PIP) [5].

Studies from the UK have shown stratified care to be superior to usual care in primary care LBP patients. Furthermore, the stratified care model resulted in a $50 \%$ reduction in LBP-related sickness absence and lower healthcare costs compared to current best practice [6]. Thus, stratified care may be associated with clinical benefits for LBP patients in primary healthcare at lower costs. However, still evidence is sparse and healthcare and social systems may vary between countries (e.g. in the way reimbursement and referral systems works). Therefore, replication of the UK findings is warranted before implementation in Danish healthcare settings can be recommended. The aim of this study is to evaluate the clinical effects and cost-effectiveness of a stratified care model in patients with NSLBP compared to current practice in a Danish primary healthcare setting.

\section{Methods, design and setting}

We will perform the study as a two-armed randomised controlled trial (RCT) in primary healthcare from December 2015 to December 2017 in the Regions of Southern and Central Denmark. Follow-up points will be at three and 12 months (for further information, see Fig. 1). All data collection and randomisation procedures will be administered through an existing web-based research portal (https://trialpartner.clin.au.dk). Patients are recruited by general practitioners (GP) in ten municipalities across Southern and Central Denmark Regions; a total of 42 GPs practices and 21 physiotherapy clinics participate. At the initial or second consultation (see Fig. 2), the GP will assess, triage and electronically refer patients to physiotherapy according to their normal practice specified in the recommendations of the Danish Society of General Practice [7]. Patients who decline referral will not be eligible for the study and will follow the usual clinical trajectory.

\section{Inclusion and exclusion criteria}

The inclusion criteria are: are patients diagnosed with NSLBP and found relevant for referral to physiotherapy by the GP; age 18 years and above; and understand Danish language. Patients with or without contributing leg pain can be included in the study.
The exclusion criteria are: serious pathology (malignancy, inflammatory arthritis, etc.); serious nerve root compression (cauda equine, paresis <3), influential co-morbidity; psychiatric illness; personality disorder; spinal surgery during the last six months; pregnancy; or already receiving physiotherapy treatment for the present episode.

\section{Recruitment \\ Clinicians}

The recruitment of GPs and clinicians will be from the same local area and cover different geographical parts of the Regions of Southern and Central Denmark. Local information meetings will be held at GP practices and the physiotherapists will be trained/instructed to deliver either stratified care (intervention) or current practice (control).

\section{Patients}

Participating patients are recruited when consulting their GP for an episode of LBP. The GP assesses and diagnoses/triages the patient according to their clinical guideline. Patients diagnosed with NSLBP and who are eligible for the project according to the inclusion and exclusion criteria are asked for their interest in participation. If interested, the patients will be handed written information and asked for their phone number for later contact. Finally, they are electronically referred to physiotherapy (via the usual electronic referral site 'RefHost'). The project secretary is continuously informed of new potential participants by the Trial partner system.

Within 1-2 days, interested patients are contacted by the project secretary by telephone. The project secretary repeats information on study details, checks inclusion/exclusion criteria and answers any questions regarding the written information. If the patient is still interested in participating, an email containing a hyperlink is sent on an encrypted line. When activating the link, a consent form appears to be signed with a digital signature. ${ }^{1}$ This form is automatically returned and the patient can now complete the baseline questionnaires.

\section{Randomisation}

After receiving the patient's questionnaire and signed consent, the project secretary randomises the patient into one of the two treatment arms stratified for city and SBT sub-group using computer-generated random number sequence (Fig. 2). By randomisation, the patients in the study are ensured to receive treatment based at least on best practice. After randomisation, the project secretary contacts the relevant physiotherapy clinic to ensure initiation of treatment. At three-month and 12-month follow-up, questionnaires are electronically sent to the patient. Electronic reminders are sent 


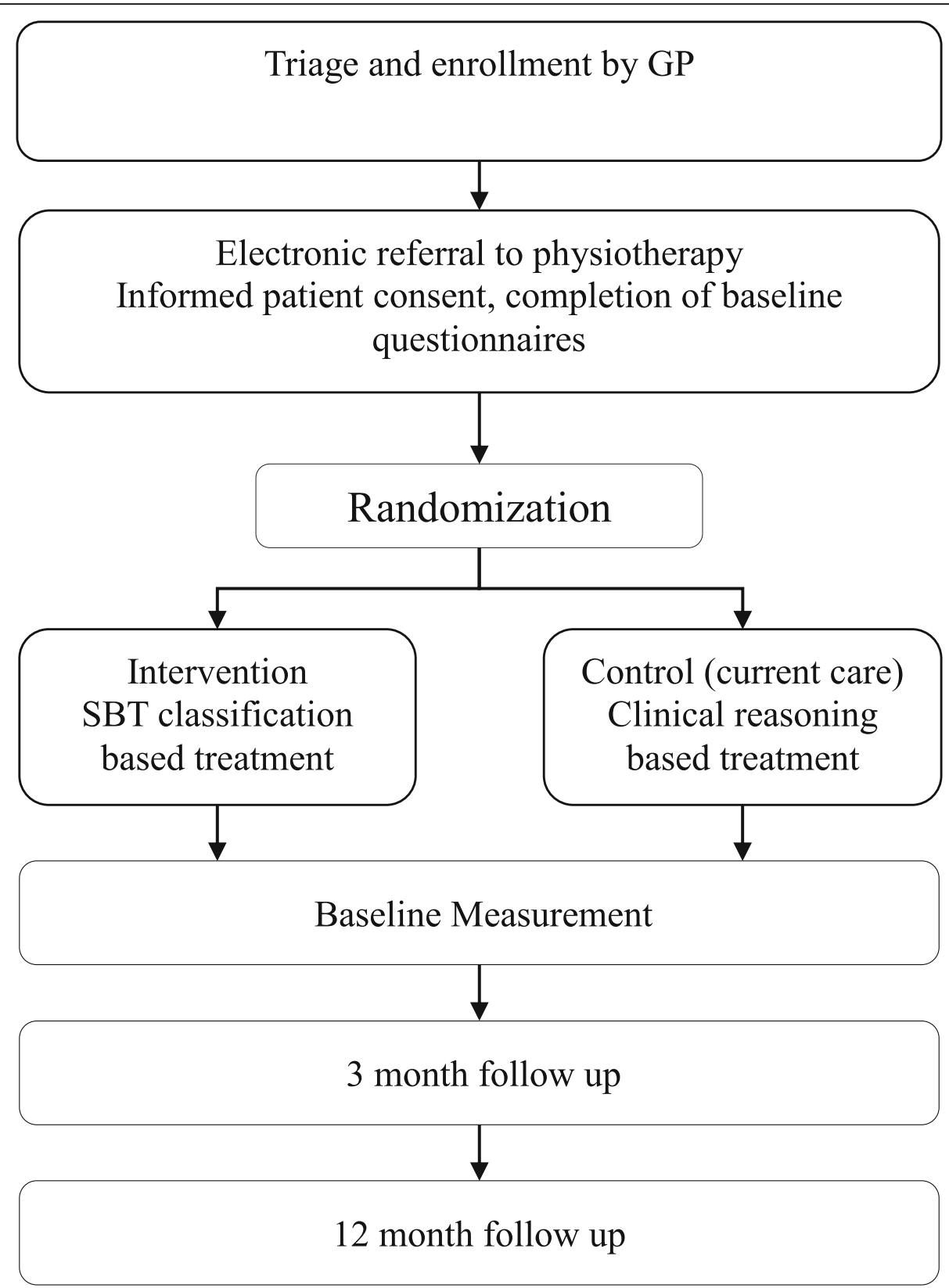

Fig. 1 Flow chart of inclusion, treatment and follow-up

after 14 days, followed by a phone reminder seven days later. Randomisation, data collection and reminders are administrated by an online clinical database developed for the study (Additional file 1).

\section{Intervention}

Stratified care group (intervention group)

A structured standardised physical assessment will be performed. This includes patient history concerns and treatment expectations and a core set of relevant physical tests (e.g. neurological examination if relevant, back pain movements and testing for a directional preference). The results of the clinical assessment, scores from questionnaires and the SBT sub-group classification in combination are used as clinical guidance and the appropriate matched treatment will be delivered accordingly. The treatment is delivered by physiotherapists who have received adequate training to deliver the stratified care. The training will consist of a five-day course. The training course includes approaches on how to address patient beliefs, attitudes and behaviour. In interactive workshops, training sessions and role plays, these approaches are 


\begin{tabular}{|c|c|c|c|c|c|c|c|c|}
\hline \multirow[b]{3}{*}{ TIMEPOINT $^{* *}$} & \multirow{3}{*}{$\begin{array}{c}\text { Enrolment } \\
-t_{1} \\
\end{array}$} & \multicolumn{7}{|c|}{ STUDY PERIOD } \\
\hline & & \multirow{2}{*}{$\begin{array}{c}\text { Allocation } \\
0 \\
\end{array}$} & \multicolumn{5}{|c|}{ Post-allocation (months) } & \multirow{2}{*}{$\begin{array}{l}\text { Close-out } \\
+12 \text { months }\end{array}$} \\
\hline & & & 0 & 3 & 6 & 9 & 12 & \\
\hline \multicolumn{9}{|l|}{ ENROLMENT: } \\
\hline \multirow{4}{*}{$\begin{array}{r}\text { Eligibility screen } \\
\text { Informed consent } \\
\text { Baseline } \\
\text { questionnaires } \\
\text { Allocation }\end{array}$} & $\mathrm{x}$ & & & & & & & \\
\hline & $x$ & & & & & & & \\
\hline & $\mathrm{X}$ & & & & & & & \\
\hline & & $\mathrm{x}$ & & & & & & \\
\hline \multicolumn{9}{|l|}{ INTERVENTIONS: } \\
\hline \multicolumn{9}{|l|}{ Stratified care } \\
\hline \multicolumn{9}{|l|}{ Current practice } \\
\hline \multicolumn{9}{|l|}{ ASSESSMENTS: } \\
\hline $\begin{array}{r}\text { Background } \\
\text { questions }\end{array}$ & $x$ & & & & & & & \\
\hline \multirow[t]{2}{*}{ STarT Back Tool* } & $\mathrm{X}$ & & & $x$ & & & & $x$ \\
\hline & $x$ & & & $x$ & & & & $\mathrm{x}$ \\
\hline $\begin{array}{r}\text { Pain } \\
\text { Roland Morris } \\
\text { Disability } \\
\text { Questionnaire }\end{array}$ & $x$ & & & $x$ & & & & $\mathrm{X}$ \\
\hline \multirow{2}{*}{$E Q-5 D$} & $X$ & & & $x$ & & & & $x$ \\
\hline & $X$ & & & $x$ & & & & $x$ \\
\hline Pain behavior & & & & $x$ & & & & $x$ \\
\hline
\end{tabular}

Fig. 2 SPIRIT. Schedule of enrolment, assessment and interventions

tested and practised. The deliverance of training will be undertaken by a highly skilled physiotherapist who was one of the developers behind the development and education in the English STarT Back Trial [8]. To ensure maintenance of skills and adherence to the protocol, clinical training and support programmes for the physiotherapists in the study intervention group will mirror the STarT Back Trial in the UK [4]. All patients receive reassuring information. The topics of reassurance are guided by the SBT score and the clinical assessment. Patients are encouraged to maintain or return to normal activity if possible. An informative back book and an online film are provided to supplement messages from the consultation. Advice and information about medication, further examinations, work, prognosis, future episodes, pain-coping strategies and individual patient concerns will be addressed. The main components of the stratified care are provided below:

Low-risk intervention: patients in the low-risk group will receive a good-quality, evidence-based consultation. Minimal intervention with emphasis on relevant information and activity will be relevant for this group. Onwards referral, investigation or further treatment is not recommended unless the physiotherapist finds it highly relevant.

Medium-risk intervention: in addition to the above, patients will receive an evidence-based standardised package of individualised treatment focusing on restoring function (targeting disability, back, leg and comorbid pain). The medium-risk group will also receive an intervention that focuses on the prevention of future back pain episodes.

High-risk intervention: in addition to the intervention described above, these patients will receive individualised PIP aiming to reduce pain and disability and improve psychological functioning (where possible). Treatment is centred around value-based goal-setting and is facilitated by the use of cognitive and/or behavioural techniques that focus on improvement in quality of life and enables patients to manage ongoing and/or future episodes of LBP [8]. 


\section{Current practice (control group)}

In contrast to the stratified care group, the decisions on whether patients should receive further physiotherapy treatment will be solely based on clinical judgement, clinical need and patient preferences. The physiotherapist has no access to any of the baseline patient questionnaires. Current best practice will be delivered by well-educated and qualified physiotherapists. We register the educational level of the physiotherapists to secure uniformity between the groups. A standardised physical assessment will be conducted. The main treatment modalities to be used should broadly reflect current practice in Danish primary care (e.g. Manual Therapy, Mechanical Diagnosis and Therapy, exercise, acupuncture, usual advice and reassurance).

\section{Outcome measures}

A core set of standardised and internationally recommended outcome measures will be applied in the trial [9]. The primary outcomes in the trial will be improvement in LBP disability measured by the Roland Morris Disability Questionnaire (RMDQ) [10], group differences in time off work and patient-reported global change. Time off work is considered a complicated measure [2], but standardised patient-reported data from the project database allows us to monitor short-term sick leave (measured in days) and information from the Danish National Register on Public Transfer Payments (DREAM) makes it possible to monitor long-term sick leave ( $>2$ weeks of consecutive absence) and other related social benefits such as pensions [11]. Secondary outcomes will be pain intensity, patient satisfaction, data on patient healthcare resource utilisation and EQ-5D-5 L $[12,13]$.

It is furthermore planned to monitor the stratified model's effectiveness on appropriateness of referrals and the ability to reduce referrals to secondary care. The outcome measures here will be numbers of referrals to secondary care using the stratified model compared to current practice, increased detail and usefulness of referrals sent to secondary care, and numbers of consultations in secondary care for patients initially exposed to stratified care compared to current practice. Data for these analyses will come from the two central secondary care spine centres in the regions.

\section{Analyses}

Analysis will be conducted using intention-to-treat. Scores of primary and secondary continuous outcomes are compared between groups using appropriate descriptive statistics, mixed multi-level linear models and Generalised Estimating Equations models for categorical outcomes at three and 12 months taking into account cluster effects of the treating physiotherapist [14]. Secondary analysis using multiple imputation on missing data and loss to follow-up will be conducted $[15,16]$. The health economic analysis will use a decision analytic framework (e.g. Markov-model or/and Decision Tree analysis) to test the cost-effectiveness of stratified care against current practice. We use a healthcare perspective for the cost-effectiveness analysis. Cost data comprise trial patient healthcare-resource use and unit cost from national provider agreements and diagnostic-related groups. Quality-adjusted life years (QALY) are calculated based on the EQ-5D with Danish QALY weights. Relevant sensitivity analyses will be performed to address the robustness of the results. A Budget Impact analysis will be made to describe the budget cost or potential budget savings for the different healthcare payers of a fully implemented SBT in the Regions compared to current practice.

The results of the study will be published according to the Consolidated Standards of Reporting Trials (CONSORT) statement for RCTs international peer-reviewed journals.

\section{Sample size}

In the UK, the STarT Back Trial protocol [17] sample size was calculated on basis of the RMDQ as the primary outcome measure. A difference of 2.5 in change scores on the RMDQ was considered to be a minimum clinically important difference. In this study, we wanted to mirror the UK study; therefore, we used the same RMDQ change score for the sample size calculation. We inserted numbers $($ mean $=13.2$, standard deviation $=5.4$ ) from a prior Danish study [3] into S-13 to calculate a sample size with a power of $80 \%$ and significance level at $5 \%$. The calculation showed that 75 patients are needed in each treatment arm to detect an overall 2.5 difference in change scores on the RMDQ between the intervention and current care group. In the study, we also want to be able to analyse the effect in the high-risk subgroup. The prevalence in Danish primary care has earlier been shown to be approximately $25 \%$. With an expected $10 \%$ drop out rate, a total of 660 patients will be included in the study.

\section{Adverse effects}

All adverse effects will be registered and reported.

\section{Discussion}

LBP constitutes a global health problem and stratified care that effectively targets treatment to relevant sub-groups of patients has the potential to have great impact on the treatment pathways of LBP. If effective, the results of this study will qualify whether stratified care models may serve as a part of the national Danish implementation strategy for evidence-based LBP care in primary healthcare. Potentially, this could significantly result in better patient outcomes and at the same time reduce the costs for treatment of LBP. 


\section{Trial status}

Recruitment is ongoing.

\section{Endnotes}

${ }^{1}$ https://www.nemid.nu/dk-da/

\section{Additional file}

Additional file 1: SPIRIT - checklist. Recommended items to address in a clinical trial protocol. (DOC $118 \mathrm{~kb}$ )

\section{Abbreviations \\ CONSORT: Consolidated Standards of Reporting Trials; DREAM: Danish National Register on Public Transfer Payments; LBP: Low back pain; NSLBP: Non-specific low back pain; PIP: Psychologically informed physiotherapy; RMDQ: Roland Morris Disability Questionnaire; SBT: STarT back tool; SPIRIT: Standard Protocol Items: Recommendations for Interventional Trials; STarT: Stratification targeted treatment}

\section{Acknowledgements}

The authors acknowledge all GPs for participation in the enrolment of patients and all physiotherapists who kept the motivation during the inclusion period. The authors also acknowledge the project secretaries and secretaries at the clinics for enrolment efforts.

\section{Funding}

The study is fully funded. The study has obtained internal funding by the Central Denmark Region and the Region of Southern Denmark and external funding from the Danish Rheumatism Association, the Association of Danish Physiotherapists and the general practitioners quality and postgraduate foundation. The funders do not have any influence in the study design, the data collection, management, analysis, the interpretation or the publication of results.

\section{Authors' contributions}

LM and DHC contributed to the conception, the overall design and the funding of the study. Both are actively involved in the conduction and analysis of the study. BSC, KRO, JS and MSJ contributed to the conception of the study. JH has contributed to the study by giving invaluable input from experiences from the UK STarT Back Trial. NBVA and FP have established a regional network of physiotherapists participating in the study. All authors have participated in critical revision of this manuscript and have read and approved the final edition.

\section{Authors' information}

LM is post-doctoral, BSC is a professor and MSJ a Ph.D. student at the Department of Regional Health Research, University of Southern Denmark, Odense. JS is a professor at the Research Unit of General Practice, University of Southern Denmark, Odense, FP and NBVA are physiotherapy primary care consultants for the regions of Southern and Central Denmark, KRO is an assisting professor at the Department of Business and Economics, COHERE, University of Southern Denmark, Odense, $\mathrm{JH}$ is an assisting professor at the Institute for Primary care and Health Sciences, Keele University, Staffordshire, UK, DHC is a post-doctoral at the Department of Occupational Medicine, Regional Hospital West Jutland, University Research Clinic, Denmark.

\section{Ethics approval and consent to participate}

Participation is based on informed consent. All patients in the study receive written and verbal information regarding the study. Consent for participation can at any time can be re-drawn and participation can be terminated without consequences for the patient. Patients who are not interested in participation in the project will be treated and referred in concordance with usual practice in primary care.

The study has been approved by the Scientific Ethics Committee of Southern Denmark (file no. S-20140205) and the Danish Data Protection Agency (file no. 15/3321). All rules of storage of personal information are met according to the Danish Data Protection Agency. Data will only be reported in anonymous form. Data management will be conducted according to the regional rules of the Region of Southern Denmark. The trial has been registered at ClinicalTrials.gov (Protocol Record S-20140205).

\section{Consent for publication \\ All authors gave consent for publication.}

Competing interests

The authors declare that they have no competing interests.

\section{Publisher's Note}

Springer Nature remains neutral with regard to jurisdictional claims in published maps and institutional affiliations.

\section{Author details}

${ }^{1}$ Centre for Quality, Region of Southern Denmark, P.V. Tuxensvej 5, 5500 Middelfart, Denmark. ${ }^{2}$ Department of Regional Health Research, University of Southern Denmark, Odense, Denmark. ${ }^{3}$ Spine Centre of Southern Denmark, Odense, Denmark. ${ }^{4}$ Research Unit of General Practice, University of Southern Denmark, Odense, Denmark. ${ }^{5}$ Department for Health Provision, Region of Central Denmark, Viborg, Denmark. ${ }^{6}$ Physiothery Primary Care Consultant, Region of Southern Denmark, Vejle, Denmark. 'Department of Business and Economics, COHERE, University of Southern Denmark, Odense, Denmark. ${ }^{8}$ Institute for Primary Care and Health Sciences, Keele University, Staffordshire, UK. ${ }^{9}$ Department of Occupational Medicine, Regional Hospital West Jutland, University Research Clinic, Herning, Denmark.

Received: 4 October 2017 Accepted: 11 May 2018

Published online: 08 June 2018

\section{References}

1. Hill JC, Whitehurst DG, Lewis M, Bryan S, Dunn KM, Foster NE, et al. Comparison of stratified primary care management for low back pain with current best practice (STarT back): a randomised controlled trial. Lancet. 2011;378(9802):1560-71.

2. Foster NE, Hill JC, O'Sullivan P, Hancock M. Stratified models of care. Best Pract Res Clin Rheumatol. 2013;27(5):649-61.

3. Morso L, Kent P, Albert HB, Hill JC, Kongsted A, Manniche C. The predictive and external validity of the STarT back tool in Danish primary care. Eur Spine J. 2013;22:1859-67.

4. Morso L, Kent P, Manniche C, Albert HB. The predictive ability of the STarT back screening tool in a Danish secondary care setting. Eur Spine J. 2014;23:120-8.

5. Hill JC, Dunn KM, Lewis M, Mullis R, Main CJ, Foster NE, et al. A primary care back pain screening tool: identifying patient subgroups for initial treatment. Arthritis Rheum. 2008;59(5):632-41.

6. Foster NE, Mullis R, Hill JC, Lewis M, Whitehurst DG, Doyle C, et al. Effect of stratified care for low back pain in family practice (IMPaCT Back): a prospective population-based sequential comparison. Ann Fam Med. 2014; 12(2):102-11.

7. Danske R: Rygomrtdet retningslinjer for visitation. 2012.

8. Main CJ, Sowden G, Hill JC, Watson PJ, Hay EM. Integrating physical and psychological approaches to treatment in low back pain: the development and content of the STarT back trial's 'high-risk' intervention (StarT back; ISRCTN 37113406). Physiotherapy. 2012;98(2):110-6.

9. Chapman JR, Norvell DC, Hermsmeyer JT, Bransford RJ, DeVine J, McGirt MJ, et al. Evaluating common outcomes for measuring treatment success for chronic low back pain. Spine (Phila Pa 1976). 2011;36(21 Suppl):S54-68.

10. Roland M, Fairbank J. The Roland-Morris disability questionnaire and the Oswestry disability questionnaire. Spine (Phila Pa 1976). 2000;25(24):3115-24.

11. Hjollund NH, Larsen FB, Andersen JH. Register-based follow-up of social benefits and other transfer payments: accuracy and degree of completeness in a Danish interdepartmental administrative database compared with a population-based survey. Scand J Public Health. 2007;35(5):497-502.

12. Brooks R. EuroQol: the current state of play. Health Policy. 1996;37(1):53-72.

13. Janssen MF, Pickard AS, Golicki D, Gudex C, Niewada M, Scalone L, et al. Measurement properties of the EQ-5D-5L compared to the EQ-5D-3L across eight patient groups: a multi-country study. Qual Life Res Int J Qual Life Asp Treat Care Rehab. 2013;22(7):1717-27.

14. Twisk J. Applied longitudinal data analysis for epidemiology: a practical guide. 2nd ed. New York: Cambridge University Press; 2013. 
15. Donders AR, van der Heijden GJ, Stijnen T, Moons KG. Review: a gentle introduction to imputation of missing values. J Clin Epidemiol. 2006;59(10):1087-91.

16. van der Heijden GJ, Donders AR, Stijnen T, Moons KG. Imputation of missing values is superior to complete case analysis and the missingindicator method in multivariable diagnostic research: a clinical example. J Clin Epidemiol. 2006;59(10):1102-9.

17. Hay EM, Dunn KM, Hill JC, Lewis M, Mason EE, Konstantinou K, et al. A randomised clinical trial of subgrouping and targeted treatment for low back pain compared with best current care. The STarT back trial study protocol. BMC Musculoskelet Disord. 2008;9:58.

- fast, convenient online submission

- thorough peer review by experienced researchers in your field

- rapid publication on acceptance

- support for research data, including large and complex data types

- gold Open Access which fosters wider collaboration and increased citations

- maximum visibility for your research: over $100 \mathrm{M}$ website views per year 